\title{
FPGA-based lens undistortion and image rectification for stereo vision applications (Erratum)
}

Christina Junger, Albrecht Hess, Maik Rosenberger, Gunther Notni

Christina Junger, Albrecht Hess, Maik Rosenberger, Gunther Notni, "FPGAbased lens undistortion and image rectification for stereo vision applications (Erratum)," Proc. SPIE 11144, Photonics and Education in Measurement Science 2019, 111441F (17 February 2021); doi: 10.1117/12.2595882

SPIE Event: Joint TC1 - TC2 International Symposium on Photonics and Education in Measurement Science 2019, 2019, Jena, Germany 


\section{FPGA-based lens undistortion and image rectification for stereo vision applications (Erratum)}

Christina Junger, Albrecht Heß, Maik Rosenberger, Technische Univ. Ilmenau (Germany); Gunther Nołni, Technische Univ. Ilmenau (Germany) and Fraunhofer Institute for Applied Optics and Precision Engineering (Germany)

Proceedings Volume 11144, Photonics and Education in Measurement Science 2019; 1114416 (2019) https://doi.org/10.1117/12.2530692

Event: Photonics and Education in Measurement Science 2019, Jena, Germany

Online Publication Date: 17 September 2019

Erratum Published: 17 February 2021

A revised version of this manuscript was published on 17 February 2021. Details of the revision are provided in the text that accompanies this Erratum. The original paper has been updated. 